\title{
Neural Network Based Modeling for Polycaprolactone Synthesis by Bio-Polymerization of $\varepsilon$-caprolactone
}

\author{
Senthil Kumar Arumugasamy, M. H. Uzir, and Z. Ahmad
}

\begin{abstract}
-xtensive study of ring-opening polymerization $\varepsilon$-caprolactone ( $\varepsilon$-CL) using lipase Novozym 435 (immobilized form of lipase $B$ from Candida antarctica) as biocatalyst using ring-opening polymerization (ROP) of E-caprolactone was carried out at impeller speeds of $250,500,750,1000 \mathrm{rpm}$ and temperature of the reactor of $60^{\circ} \mathrm{C}, 70^{\circ} \mathrm{C}$ and $80^{\circ} \mathrm{C}$. The maximum molecular weight out of all the experiments carried out is $\mathbf{3 1 0 0 0 0}$ Kilo Daltons (weight average molecular weight, Mw) which was obtained at a temperature of $70^{\circ} \mathrm{C}$ and 3 hours for an impeller speed of $500 \mathrm{rpm}$. In order to develop a predictive model a multilayer feed-forward neural network (FANN) trained with an error back-propagation algorithm was incorporated. The results showed that a 3-7-1 for FANN1 with the inclusion of the Reactor impeller speed and 2-6-1 FANN2 arrangement with exclusion of the Reactor impeller gave the best performance.
\end{abstract}

Index Terms-Biopolymers, enzymatic polymerization, molecular weight distribution, polycaprolactone synthesis, ring opening polymerization (ROP)

\section{INTRODUCTION}

Enzymatic polymerization as the name suggests is the polymerization with enzyme as the catalyst. Enzymatic ROP is a form of transesterification. Here, cyclic esters (lactones) are used as substrate and are opened by the enzyme. Of all available lipases, by far the most used in organic chemistry is Candida Antarctica Lipase B (CALB). Enzyme catalyzed lactones ROP in organic media have been conducted for the polymerizations of $\varepsilon$-caprolactone $(\varepsilon-C L)[1,2]$. Recently several researches have been carried out in this field from creating new biopolymers with the exisiting lipases to synthesizing biopolymers using newly developed enzymes. To name a few Kobayashi et al [3] created novel biodegradable and biobased thermoplastic elastomers by the copolymerization of various macrolides and methyl $12 \mathrm{HS}$ (12HS-Me) using lipase as a catalyst Sandoval et al [4] used the extracellular lipase from the yeast Yarrowia lipolytica immobilized on hydrophobic supports and also used the auto-immobilized lipase from agro-wastes from Carica papaya as a new cheap and efficient biocatalyst for biopolymer synthesis. Liu et al [5] utilized the lipase from Candida sp.99-125 firstly for polymerization of diethyl sebacate and 1, 4-butanediol in absence of organic solvents, concentrating on yielding poly (butylene sebacate) of high molecular weight. Ma Et al [6] reported the ring-opening

Manuscript received September 21, 2012; revised November 28, 2012. This work was supported by research funding from School of Chemical Engineering, Universiti Sains Malaysia

Senthil Kumar Arumugasamy, M. H. Uzir, and Z. Ahmad are with Universiti Sains Malaysia (e-mail: senthilkumar9999@gmail.com). polymerization of -caprolactone catalyzed by thermophilic esterase Archaeoglobus fulgidus (AFEST). Few researchers have also worked on the kinetic studies and also in developing mathematical models. To name a few Lipik et al [7] synthesized a series of polymers, according to experimental design and developed a mathematical model, which can account for the real molar mass of a synthesized polymer using the main polymerization parameters as the variables. Hence the objective of this paper was to model the large scale production of polycaprolactone determining the effect of temperature, impeller speed. In this study feed forward neural network (FANN) model of the biopolymerization process was developed. Two FANN models were obtained one with the inclusion of the reactor impeller speed and other with its exclusion as an input.

\section{MATERIALs AND Methods}

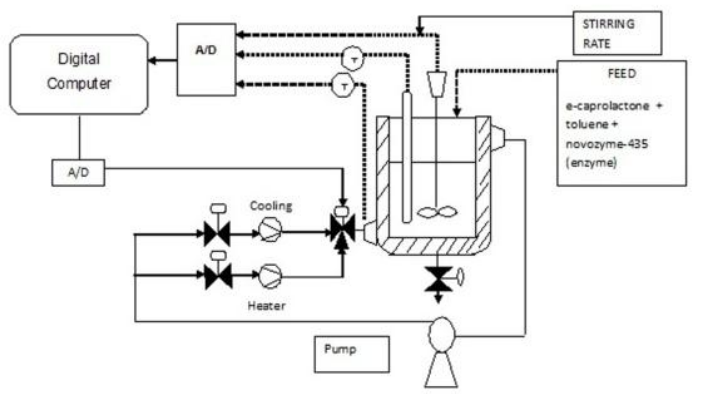

Fig. 1. Schematic diagram of batch bio-reactor

Polymerization grade $\varepsilon$-caprolactone purchased from Merck private limited, was first dried over calcium hydride and then distilled under reduced pressure in nitrogen atmosphere. Chloroform and toluene, were purchased from Merck private limited. Toluene was dried over calcium hydride, and distilled under nitrogen atmosphere. Novozyme-435 Candida Antartica lipase (specified activity $7000 \mathrm{PLU} / \mathrm{g}$ ) was purchased from Science Technics private limited. All liquid chemical transfers were performed by syringe through rubber septum caps under nitrogen atmosphere.The ratio of solvent to monomer to catalyst $2: 1: 10(\mathrm{v} / \mathrm{v} / \mathrm{wt})$. The scale up production of polycaprolactone from e-caprolactone was carried out using a 2 litre (effective volume) Infors-HT Labfors bioreactor. Two important parameters namely temperature and reactor impeller speed were considered for the study. The details apparatus is shown in Fig. 1. The reactor was started and the reactor temperature was allowed to rise to the desired value. The stirrer was started and set to the desired speed. The mixture started to react and the samples were removed for a time interval of $1 \mathrm{hr}$ uptill 7th hr. Zeta-sizer analyser was used for the molecular 
weight analysis for this research which was performed using Zeta-sizer 1000 HS, Malvern Instruments Ltd., Worcestershire, United Kingdom (UK).

\section{FEED ForWARD NEURAL NETWORK (FANN) MODELING}

In this study, networks with fixed identical structure were developed and trained by the Levenberg-Marquardt optimisation algorithm The neural networks are single hidden layer feed forward neural networks. All simulation works are carried out using MatlabTM R2011. In order to determined the number of hidden nodes in the hidden layer, neural networks with different numbers of hidden neurons were trained on the training data and tested on the testing data. The network with the lowest sum square error (SSE) on the testing data was considered as having the best network topology. In assessing the developed models, MSE and $r$ value on the unseen validation data is used as the performance criterion. The static models for neural network development are shown below:

FANN1 (with reactor impeller speed)

$$
Y(t)=f n[u 1(t) u 2(t), u 3(t)]
$$

where:

$$
\begin{aligned}
& u 1(t)=\text { temperature } \\
& u 2(t)=\text { impeller speed } \\
& u 3(t)=\text { reaction time }
\end{aligned}
$$

FANN2 (without reactor impeller speed)

$$
Y(t)=f n[u 1(t) u 2(t)]
$$

where:

$u 1(t)=$ impeller speed

$u 2(t)=$ reaction time

\section{RESUlTS AND DISCUSSION}

\section{A. Temperature Effects}

Fig. 2 shows the variation of molecular weight with reaction time at $70^{\circ} \mathrm{C}$ with a volume ratio of $1: 2$ of $\varepsilon$-caprolactone/toluene being taken shows that $500 \mathrm{rpm}$ impeller speed is most suitable for the process as the value of molecular weight is maximum as high as $310000 \mathrm{KDa}$ (weight average molecular weight, $\mathrm{Mw}$ ) compared to that of the molecular weights obtained for $250 \mathrm{rpm}, 750 \mathrm{rpm}$ and $1000 \mathrm{rpm}$. Looking at the trend all the impeller speeds indicate the same trend of maintaining a lower value of molecular weight at the initial reaction time up till $3 \mathrm{hrs}$ after which there is an increase to be followed by a decrease in the molecular weight at the completion of the reaction nearing 7 th hrs.

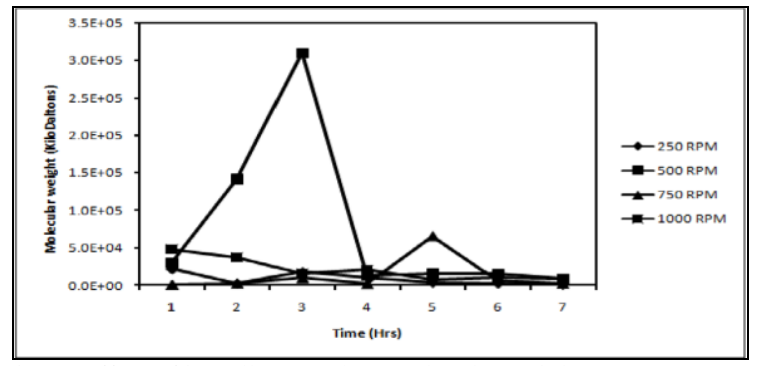

Fig. 2. Effect of impeller speed on molecular weight at a constant temperature of $70^{\circ} \mathrm{C}$

\section{B. Mixing effect}

Fig. 3 shows the effect on molecular weight at a particular impeller speed of $250 \mathrm{rpm}$, the trend followed is the same which indicates that as the reaction starts the process of polymerization at all the temperatures considered namely $60^{\circ} \mathrm{C}, 70^{\circ} \mathrm{C}, 80^{\circ} \mathrm{C}$ is found to be maximum initially at the $1 \mathrm{st}$ $\mathrm{hr}$ but as the time proceeds the molecular weight is found to decrease steadily.

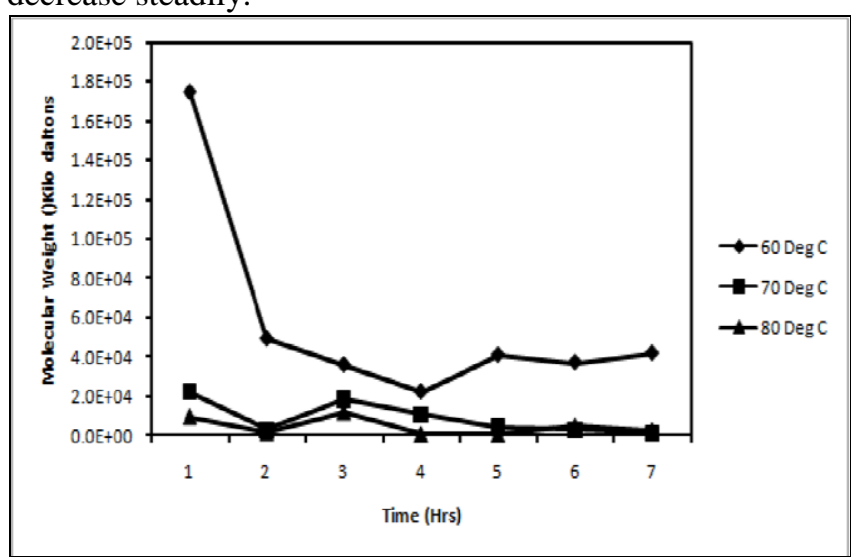

Fig. 3. Effect of Temperature on Molecular weight at of 250 RPM impeller speed

C. Molecular Weight Prediction Using Feed Forward Neural Network (FANN)

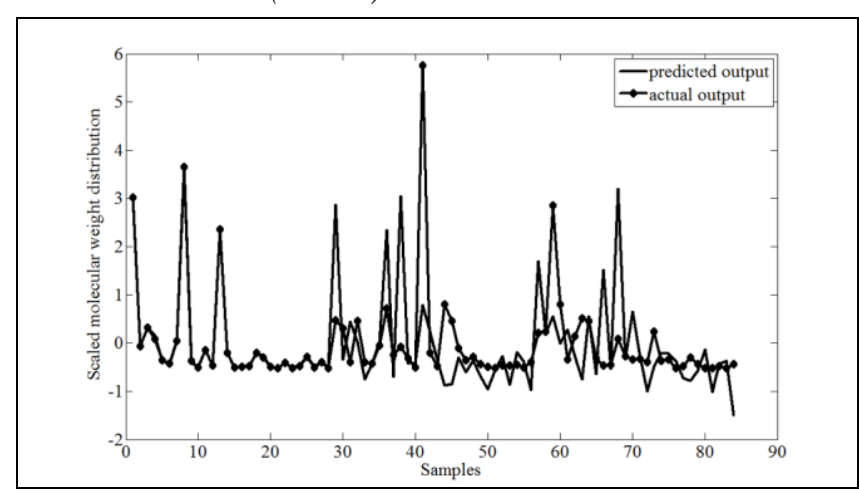

Fig. 4. Plot comparing predicted output with the actual output with reactor impeller speed

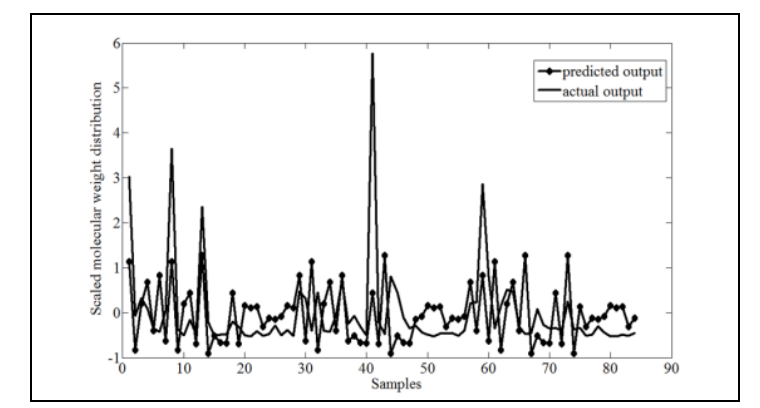

Fig. 5. Plot comparing predicted output with the actual output without reactor impeller speed

Fig. 4, 5 show the plot of ANN predicted molecular weight against the experimental output which clearly shows that the predicted molecular weight in case of experimental data with reaction time, reaction temperature along with reactor impeller speed shows better results in comparison to the results obtained by excluding the reactor impeller speed. Figure 6 indicated the residue plot which is the difference between the actual data and the predicted data for the reactor study with and without reactor impeller speed. The 
discrepancies between the actual and predicted data are very small. Fig. 6 once again proves that the residue where inreactor impeller speed is taken into account proves better rather than excluding the reactor impeller speed.

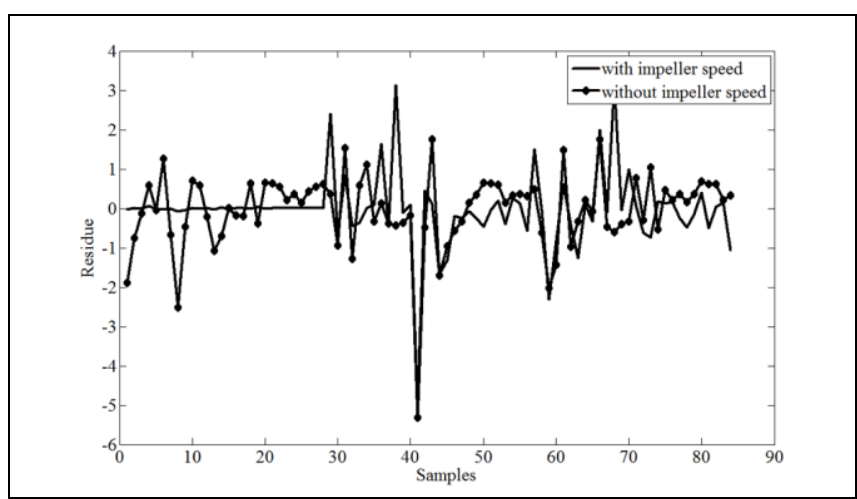

Fig. 6. Comparison of residue plot for the model with and without reactor impeller speed.

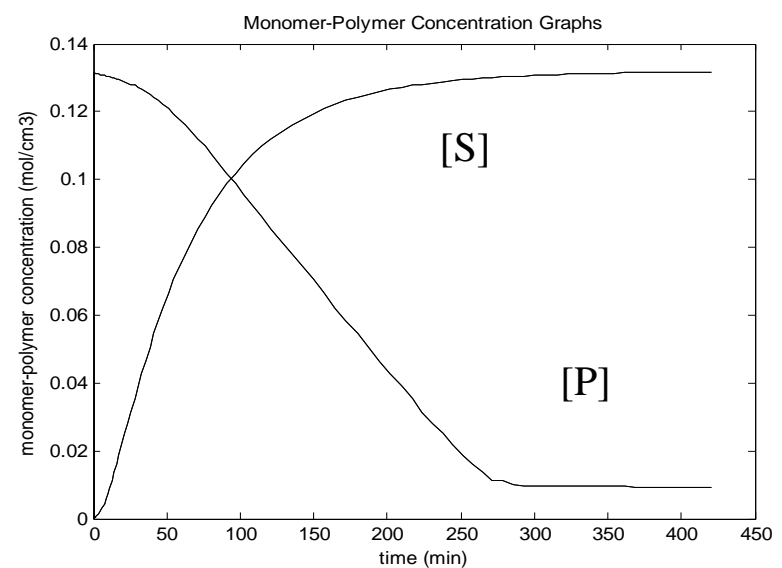

Fig. 7. Monomer (substrate)-polymer concentration plot from mechanistic model

Mathematical model or first principle model is a model that is developed based on the knowledge of the desired process. Therefore, verification process acts as a platform to prove the validity of the model. The nature of biopolymerization process suggests that the product (biopolymer) increased as the monomer is consumed. In this work, enzymatic polymerization of $\varepsilon$-caprolactone is perceived to follow this enzyme-catalyzed reaction behavior. In the graph, $[\mathrm{P}]$ represents the curve for the product while $[\mathrm{S}]$ shows the substrate's trend in the reaction.

SSE, RMSE and R values are the renowned method of assessment for neural network model. The values of the above mentioned parameters can be seen in the Table 1 . In this work, one-way ANOVA is employed to the FANN1 and FANN2. One-way ANOVA demonstrates the relation between mean of the reactor level study with and without impeller speed. Hence the standard ANOVA table determines the sums of squares, degrees of freedom, mean squares (SS/df), F statistic, and p value. p-value from the ANOVA results for FANN1 and FANN2 are 0.8187 and 0.7216 respectively. Based on the results, the means for the reaction displays significantly different means (significant results are signified as less than 0.05 or 0.01 ). This is because results show a slight difference molecular weight between reactor and neural network model. Nevertheless, the results exhibit the same molecular weight trend.

TABLE I: CORRELATION COEFFICIENT VALUE AND MSE

\begin{tabular}{|l|c|c|l|c|}
\hline Data & \multicolumn{2}{|l|}{ NN1(with impeller speed) } & $\begin{array}{l}\text { NN2(without } \\
\text { speed) }\end{array}$ & MSE \\
\cline { 2 - 5 } & $\begin{array}{l}\text { Correlation } \\
\text { Coefficient, } \boldsymbol{r}\end{array}$ & MSE & $\begin{array}{l}\text { Correlation } \\
\text { Coefficient, } \boldsymbol{r}\end{array}$ & 0.867 \\
\hline Training & 0.998 & 0.0052 & 0.550 & 0.099 \\
\hline Testing & 0.799 & 0.9681 & 0.757 & 0.064 \\
\hline Validation & 0.633 & 3.0859 & 0.264 & \\
\hline
\end{tabular}

\section{CONCLUSION}

In this paper the effects of temperature and mixing on ring-opening polymerizations (ROP) of $\varepsilon$-caprolactone was studied by using lipase enzyme Novozym 435 (immobilized form of lipase B from Candida antarctica) as biocatalyst where in the maximum molecular weight out of all the experiments carried out is $310000 \mathrm{KDa}$ at $70^{\circ} \mathrm{C}, 3$ hours and $500 \mathrm{rpm}$ impeller speed. The conclusion is that $500 \mathrm{rpm}$ is found to be the most appropriate speed for the impeller that needs to be maintained for the enzymatic catalyzed polymerization process. The results of modeling study showed that FANN1 outperformed FANN2.

\section{ACKNOWLEDGEMENT}

This work was supported by the Universiti Sains Malaysia (USM) Research University (RUI) Grant (USM-RUI) 814076 .

\section{REFERENCES}

[1] D. Knani, A. L. Gutman, and D. H. Kohn, "Enzymatic polyesterification in organic media. Enzyme-catalyzed synthesis of linear polyesters. I. Condensation polymerization of linear hydroxyesters. II. Ring-opening polymerization of $\epsilon$-caprolactone," $J$. Polym. Sci. Part A Polym. Chem, vol. 31, no. 5, pp. 1221-1232, 1993.

[2] R. T. MacDonald, S. Pulapura, Y. Y. Svirkin, R. A. Gross, D. L. Kaplan, J. Akkara, and G. Swift, "Enzyme catalyzed $\varepsilon$-caprolactone ring-opening polymerization," Macromolecules, vol. 28, pp. 73-78, 1995.

[3] T. Kobayashi and S. Matsumura, "Enzymatic synthesis and properties of novel biodegradable and biobased thermoplastic elastomers," Polymer Degradation and Stability, vol. 96, no. 12, pp. 2071-2079, 2011.

[4] G. Sandoval, I. Rivera, K.A. Barrera-Rivera, and A. Martı'nez-Richa, "Biopolymer Synthesis Catalyzed by Tailored Lipases," Macromol. Symp., vol. 289, no. 1, pp. 135-139, 2010.

[5] W. Liu, B. Chen, F. Wang, and T. Tan, "Lipase-catalyzed synthesis of aliphatic polyesters and properties characterization," Process Biochemistry, vol. 46, no. 10, pp. 1993-2000, 2011.

[6] J. Ma, Q. Li, B. Song, D. Liu, B. Zheng, Z. Zhang, and Y. Feng, "Ring-opening polymerization of $\varepsilon$-caprolactone catalyzed by a novel thermophilic esterase from the archaeon Archaeoglobus fulgidus," Journal of Molecular Catalysis B: Enzymatic, vol. 56, no. 2-3, pp. 151-157, 2009.

[7] V. T. Lipik and M. J. M. Abadie, "Process Optimization of Poly (ع-caprolactone) Synthesis by Ring Opening Polymerization," Iranian Polymer Journal, vol. 19, no. 11, pp. 885-893, 2010. 